2017-04-01

\title{
Motor Preparation Rather Than Decision-Making Differentiates Parkinson's Disease Patients With And Without Freezing of Gait
}

John Butler

Technological University Dublin, john.s.butler@tudublin.ie

Conor Fearon

Trinity College Dublin, Ireland

Isabelle Killane

Technological University Dublin, isabelle.killane@tudublin.ie

See next page for additional authors

Follow this and additional works at: https://arrow.tudublin.ie/scschmatart

Part of the Mathematics Commons

\section{Recommended Citation}

Butler, J. et al. (2017) Motor Preparation Rather Than Decision-Making Differentiates Parkinson's Disease Patients With And Without Freezing of Gait. Clinical Neurophysiology, Volume 128, Issue 3, March 2017, Pages 463-471. doi:10.1016/j.clinph.2016.12.019

This Article is brought to you for free and open access by the School of Mathematics at ARROW@TU Dublin. It has been accepted for inclusion in Articles by an authorized administrator of ARROW@TU Dublin. For more information, please contact arrow.admin@tudublin.ie, aisling.coyne@tudublin.ie,gerard.connolly@tudublin.ie.

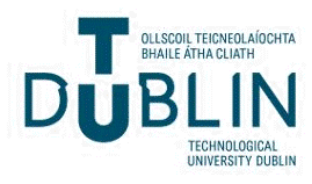




\section{Authors}

John Butler, Conor Fearon, Isabelle Killane, Saskia M. Waechter, Richard B. Reilly, and Timothy Lynch 


\section{Accepted Manuscript}

Motor Preparation Rather Than Decision-Making Differentiates Parkinson's Disease Patients With And Without Freezing of Gait

John S. Butler, Conor Fearon, Isabelle Killane, Saskia M. Waechter, Richard B. Reilly, Timothy Lynch

PII:

S1388-2457(16)31031-8

DOI: http://dx.doi.org/10.1016/j.clinph.2016.12.019

Reference: CLINPH 2008018

To appear in:

Clinical Neurophysiology

Received Date:

21 July 2016

Revised Date:

4 December 2016

Accepted Date:

18 December 2016

Please cite this article as: Butler, J.S., Fearon, C., Killane, I., Waechter, S.M., Reilly, R.B., Lynch, T., Motor Preparation Rather Than Decision-Making Differentiates Parkinson's Disease Patients With And Without Freezing of Gait, Clinical Neurophysiology (2016), doi: http://dx.doi.org/10.1016/j.clinph.2016.12.019

This is a PDF file of an unedited manuscript that has been accepted for publication. As a service to our customers we are providing this early version of the manuscript. The manuscript will undergo copyediting, typesetting, and review of the resulting proof before it is published in its final form. Please note that during the production process errors may be discovered which could affect the content, and all legal disclaimers that apply to the journal pertain. 


\section{Motor Preparation Rather Than Decision-Making Differentiates Parkinson's Disease Patients With And Without Freezing of Gait}

John S. Butler*1,2,3, Conor Fearon*1,2,4 Isabelle Killane ${ }^{1,2}$, Saskia M. Waechter ${ }^{1,2}$, Richard B. Reilly ${ }^{1,2,5}$, Timothy Lynch ${ }^{4}$

${ }^{1}$ Trinity Centre for Bioengineering, Trinity College, The University of Dublin, Dublin 2, Ireland

${ }^{2}$ School of Engineering, Trinity College, The University of Dublin, Dublin 2, Ireland ${ }^{3}$ School of Mathematical Sciences, Dublin Institute of Technology, Kevin Street, Dublin, Ireland

${ }^{4}$ Dublin Neurological Institute at the Mater Misericordiae University Hospital, Dublin 7, Ireland

${ }^{5}$ School of Medicine, Trinity College, The University of Dublin, Dublin 2, Ireland

\section{Corresponding authors:}

- Timothy Lynch, Dublin Neurological Institute at the Mater Misericordiae University Hospital, 57 Eccles Street, Dublin 7, tlynch@dni.ie

- Richard B. Reilly, Trinity Centre for Bioengineering, the School of Medicine and the School of Engineering, Trinity College Dublin, Ireland, rbreilly@tcd.ie

${ }^{*}$ J. S. Butler and C. Fearon contributed equally to the paper. 


\section{Abstract}

Objective: Freezing of gait (FOG) is a brief, episodic phenomenon affecting over half of people with Parkinson's disease (PD) and leads to significant morbidity. The pathophysiology of FOG remains poorly understood but is associated with deficits in cognitive function and motor preparation.

Method: We studied 20 people with PD (10 with FOG, 10 without FOG) and performed a timed response target detection task while electroencephalographic data were acquired. We analysed the data to detect and examine cortical markers of cognitive decision making (P3b or centroparietal positivity, CPP) and motor readiness potential. We analysed current source density (CSD) to increase spatial resolution and allow identification of distinct signals.

Results: There was no difference in the $\mathrm{P} 3 \mathrm{~b} / \mathrm{CPP}$ response between people with PD with and without FOG, suggesting equivalent cognitive processing with respect to decision-making. However, the FOG group had significant difference with an earlier onset and larger amplitude of the lateralized readiness potential. Furthermore, the amplitude of the lateralised readiness potential correlated strongly with total Frontal Assessment Battery score.

Conclusions: The difference in lateralized readiness potentials may reflect excessive recruitment of lateral premotor areas to compensate for dysfunction of the supplementary motor area and resultant loss of automatic motor control. This early, excessive recruitment of frontal networks occurs in spite of equivalent motor scores and reaction times between groups.

Significance: The saturation of frontal processing mechanisms could help explain deficits in attentional set-shifting, dual-tasking and response inhibition which are frequently reported in FOG.

Keywords: Parkinson's Disease, Freezing of Gait, Decision Making, Motor Preparation, EEG, Current Source Density, Event Related Potentials. 


\section{Highlights}

- Analysis and theoretical framework allowing interpretation of decision and motor preparation signals.

- $\quad$ Differences in motor preparation potentials between PD with and without FoG but not decision signals.

- $\quad$ The amplitude of the motor preparation potential correlates with Frontal Assessment Battery scores. 


\section{Introduction:}

Freezing of gait (FOG) is a brief, episodic phenomenon, characterised by the "absence or marked reduction in forward progression of the feet despite the intention to walk" (Nutt et al., 2011). This paroxysmal symptom affects over half of people with Parkinson's disease (PD) over time (Giladi and Nieuwboer, 2008) and is closely associated with falls and admissions to nursing homes (Bloem et al., 2004). The pathophysiology of FOG remains poorly understood but freezing is closely associated with deficits in motor parameters, such as stride time, gait symmetry and rhythmicity (Killane et al., 2015) and cognitive impairment, especially, executive dysfunction (Maruyama and Yanagisawa, 2006, Amboni et al., 2008). Executive function is impaired in PD with FOG (FOG+) compared to those without FOG (FOG-). There are specific deficits in divided attention (Spildooren et al., 2010, Tard et al., 2014), set-shifting (Shine et al., 2013b), response inhibition (Cohen et al., 2014) and conflict resolution (Vandenbossche et al., 2012). Although cognitive dysfunction probably plays a significant role in its pathogenesis, objective quantitative measures of cognitive dysfunction in FOG are lacking. Neuroimaging studies in FOG cannot directly infer cognitive dysfunction and standard neurocognitive batteries such as the Montreal Cognitive Assessment (MoCA) and Frontal Assessment Battery (FAB) remain a relatively insensitive way to assess cognition. Electroencephalography (EEG) can be helpful in the study of freezing as the high temporal resolution allows accurate detection of brief neural responses detectable during paroxysms of freezing (Handojoseno et al., 2012, Thevathasan et al., 2012, Handojoseno et al., 2013, Singh et al., 2013, Shine et al., 2014, Toledo et al., 2014, Velu et al., 2014). However, no EEG study in FOG has examined decision-making tasks which require motor output.

Event-related potentials (ERPs) are EEG surface potentials generated by a psychophysiological event, often a sensory stimulus, and are electrophysiological indicators of cognitive function. The "classical" $\mathrm{P} 3 \mathrm{~b}$ potential is a large-amplitude global reference ERP with a positive peak around 300-600 msecs following a task-relevant stimulus (Sutton et al., 1965, Polich, 2007). More recently, the equivalent term "centroparietal positive potential" (CPP), generated by different analysis methods, has been used to describe this potential ( $\mathrm{O}^{\prime} \mathrm{Connell}$ et al., 2012). The precise neural substrates of the P3b/CPP are not understood. However, P3b abnormalities correlate with executive dysfunction (Kindermann et al., 2000), response conflict and response inhibition (Groom and Cragg, 2015) . All of which probably have a central role in FOG (Vandenbossche et al., 2012, Cohen et al., 2014). Recently, the P3b has also been shown to be involved in decision making in response to sensory stimuli (Twomey et al., 2015). This signal increases in amplitude as sensory information accumulates before, reaching a threshold at which a response is executed. P3b latency is increased in PD compared with healthy controls and correlates with disease severity and cognitive dysfunction (O'Donnell et al., 1987, Toda et al., 1993, Katsarou et al., 2004, Matsui et al., 2007). No study to date has examined whether differences in these measures exist between FOG + and FOG- in PD. 
ERP analysis can also be used to study the electrical correlates of motor preparation. The readiness potential, also known as the Bereitschaftpotential, is a movement-related cortical potential preceding voluntary or goal-directed movement (for a review of movement potentials in Parkinson's see (Georgiev et al., 2016)). It reflects electrical activity in the motor cortex, premotor area (PMA) and supplementary motor area (SMA) (Shibasaki and Hallett, 2006). This negative potential has to reach a certain threshold before movement or EMG activity is triggered. Readiness potentials for self-initiated, but not externally triggered, movements are attenuated in PD and correlate with reduced regional blood flow in the SMA (Jahanshahi et al., 1995). This SMA dysfunction may be compensated for by lateral premotor activation (Cunnington et al., 1995). Dysfunction of the SMA may be integral to the pathophysiology of FOG (Nutt et al., 2011), however there has been no study of readiness potentials in FOG to date.

Freezing is characterized not only by the arrest of movement but also by the initial intention to move(Nieuwboer and Giladi, 2013). For this reason, we hypothesized that motor initiation in FOG- and FOG+ will be different. Even simple motor tasks require both decision-making and motor preparation. Of note, freezing is associated with both cognitive and motor deficits. We performed an EEG-based analysis on FOG- and FOG+ to simultaneously analyse cognitive ERPs and motor readiness potentials. We hoped to deduce whether impairments in cognitive processing or motor initiation (or both) differentiates FOG- from FOG+. In order to separate the decision making and motor preparation cortical signals, we used a spatial filter known as the current source density (CSD) to increase the spatial resolution of the data. This method employs a local reference point, thus reducing interference from remote sources and current diffusions through the skull, leading to better spatial resolution compared with the global reference used in standard ERP approaches. CSD has been shown to separate these two signals in healthy participants (Kelly and O'Connell, 2013). These methods are described in detail below and we highlight their importance in ERP analysis in PD.

\section{Methods:}

\subsection{Participants:}

We recruited 20 people with PD (as defined by the UK Brain Bank Criteria (Hughes et al., 1992), Hoehn and Yahr stage II-III) from the Movement Disorder clinic at the Dublin Neurological Institute at the Mater Misericordiae University Hospital. Ethical approval was granted from the hospital ethics committee and informed consent was obtained from all participants. All patients underwent clinical and neuropsychological testing including Montreal Cognitive Assessment (MoCA), Frontal Assessment Battery (FAB) and Unified Parkinson's Disease Rating Scale III (UPDRS III). FOG status was recorded for all patients based on by observation of a movement disorder specialist and Question 1 of the New Freezing of Gait Questionnaire ("Did you experience a freezing episode over the past month?") (Nieuwboer et al., 2008). All participants had normal corrected vision and were tested in the "on"-state. 


\subsection{Stimuli and Procedure:}

Participants were seated comfortably and performed a two-stimulus oddball task consisting of a flashing green cross presented randomly on a 55" LCD monitor. This visual stimulus consisted of either vertical (standard) or $45^{\circ}$ rotated (target) green crosses presented for 500 msecs on a complex background. The standard stimulus was presented $80 \%$ of the time and the participant was instructed not to respond to this stimulus. For the remaining $20 \%$, the target stimulus was presented and participants were instructed to press the button with their right hand as soon as the target stimulus was seen. The standard and target stimuli were presented with random interstimulus intervals of between 250 and 750 msecs. A single trial of 300 seconds was performed for each participant. Participants were instructed to minimize head movements during the trial.

\subsection{Data Acquisition:}

We recorded synchronous electroencephalographic (EEG) data in all participants using a 128-channel BioSemi ActiveTwo EEG acquisition system during the task. Electrodes were placed using an adapted extension of "10-20" arrangement according to the Biosemi designed equiradial system (http://www.biosemi.com/headcap.htm) and amplified at source by the internal pre-amplifier. Data were recorded at a digitization rate of $2048 \mathrm{~Hz}$ with an open pass-band from DC to $150 \mathrm{~Hz}$. The desktop PC sent triggers (to indicate when oddball paradigm stimuli were presented) to the receiver box via a parallel cable. The EEG data and triggers were then visualised with Actiview (Biosemi) software on a separate notebook.

\subsection{Data Analysis:}

\subsubsection{Behavioral}

We acquired button press responses during the recording of the EEG and processed them offline using MATLAB (Mathworks, Natick, MA). Reaction time (time between stimulus presentation and button press response, RT) means and standard deviations were calculated for each participant. Only trials with reaction times falling within $200 \mathrm{~ms}$ and $1000 \mathrm{~ms}$ of target presentation were considered valid. As the data are from a clinical population with a hypokinetic movement disorder, significant inter- and intra-subject variability in reaction time was expected. The data were submitted to an unpaired t-test to assess group reaction time differences.

\subsubsection{EEG Analysis}

Using custom MATLAB scripts, we downsampled the continuous data to $512 \mathrm{~Hz}$ and band-pass filtered offline between 0.1 and $30 \mathrm{~Hz}$ ( $6 \mathrm{~dB} /$ octave). We then epoched the filtered data to both standard and target stimuli as well as to button press responses. This allowed examination of both stimulus-locked and response-locked ERPs. Epochs of $1000 \mathrm{~ms}$ with $200 \mathrm{~ms}$ pre-stimulus were extracted from the data. An automatic artifact rejection criterion of $\pm 100 \mu \mathrm{V}$ was applied across all electrodes in the array, and channels with a standard deviation of $<0.5 \mu \mathrm{V}$ were rejected. We rejected trials with more than 5 artifact channels. In trials with less than 5 such channels, any remaining bad channels were interpolated using the nearest neighbor spline (Perrin et al., 1989). The epochs 
were baseline corrected with respect to $200 \mathrm{~ms}$ pre-stimulus period. Average responses were calculated for each group to assess for the presence of betweengroup differences in amplitude of the components.

To increase spatial selectivity and minimize volume conduction the ERP data were converted using a Laplacian transformation to calculate the second spatial derivative of the potentials known as the current source density (CSD) (Perrin et al., 1989), with the units microvolts per meter squared $\left(\mu \mathrm{V} / \mathrm{m}^{2}\right)$. The matlab CSD toolbox was used to compute the scalp surface Laplacian (Kayser and Tenke, 2006). This step was introduced to improve spatial resolution in order to better discriminate between frontocentral motor preparation signals and centroparietal decision-making signals (Kelly and O'Connell, 2013). Separate plots and averages were generated for responses to the target stimulus and to the standard stimulus.

To investigate decision making, activity over central parietal ( $\mathrm{CPz}$ ) area was chosen to represent the stimulus locked target and standard P3/CPP component, indicated by the three electrode locations (blue dots corresponding to electrodes A2, A3 and A4 in the 128 Biosemi ABC electrode layout) in the head schematic in Figure 1 and Figure 2. To investigate unimanual motor preparation, a lateralized readiness potential (LRP) was calculated by subtracting the activity over the left frontocentral (FC4 corresponding to electrodes D3, D4 and D5 in the 128 Biosemi ABC electrode layout) scalp from the right frontocentral (FC3 corresponding to electrodes C3, C4 and C5 in the 128 Biosemi ABC electrode layout) scalp indicated by the electrode locations in red and green dots, respectively in the head schematic in Figure 2. Given the dense recording montage for the planned comparisons and figures, each site of interest is represented by an average of the three nearest electrodes. This process serves to increase the signal-to-noise ratio.

For the stimulus-locked conditions, the average peak amplitude was encapsulated by a $200 \mathrm{msec}$ time window around the mean group reaction time of 554ms. Group-related differences in the P3/CPP mean amplitude (suggesting group differences in decision making) were statistically assessed by two-way repeated measures ANOVA with factors of group (FOG-, FOG+) and condition (Target and Standard). Greenhouse-Geisser corrections were applied when appropriate. Group-related differences in the LRP amplitude were statistically assessed by unpaired t-tests. To test for differences in the LRP onset between groups (suggesting group differences in motor preparation) unpaired t-tests were conducted at each time point. To control for Type I errors a period of statistical significance was only considered if an alpha criterion of 0.05 or less was obtained for at least, $\sim 21 \mathrm{~ms}, 11$ consecutive time points (Guthrie and Buchwald, 1991).

\subsubsection{Regression Analysis}

There is a close association between executive dysfunction and FOG. To explore the relationship between the electrophysiological marker of motor preparation (the LRP) and the Frontal Executive Battery score, a regression analysis was performed on the entire PD group. An important confounder in many FOG 
studies is disease duration. Therefore, the multiple linear regression was calculated to predict the LRP amplitude based on the patients total FAB score and disease duration.

\subsubsection{Bayes Factor Analysis}

The Bayes factor analysis provides a measure of evidence for one model versus another (Dienes, 2016), here it is used to investigate evidence for the null hypothesis (that there is no difference in PD with and without FOG) or the alternative hypothesis (that there is a difference in PD with and without FOG). The JZS Bayes factor was computed using the R package BayesFactor using the default effect size of 0.707 (Rouder et al., 2009). A JZS Bayes factor can be read such that a JZS Bayes factor greater than 1 favours the null hypothesis over the alternative hypothesis, while a JZS Bayes factor less than 1 is the opposite.

\section{Results:}

\subsection{Demographics}

The demographic and neurocognitive data for the PD cohort (divided by FOG status) is given in Table 1.

\begin{tabular}{lll}
\hline & FOG- & FOG+ \\
\hline N & 10 & 10 \\
Age (years) & $62.5(7.9)$ & $65.3(7.6)$ \\
Gender (M:F)* & $4: 6$ & $8: 2$ \\
H\&Y stage (median) & $2.3(0.35)$ & $2.6(0.37)$ \\
Disease Duration (years)* & $7.0(3.6)$ & $13.5(9.1)$ \\
UPDRS & $29.1(14)$ & $28.3(9.7)$ \\
MOCA & $26.1(2.9)$ & $24.3(2.9)$ \\
FAB* & $17.3(1.3)$ & $15.2(2.6)$ \\
\hline
\end{tabular}

Table 1. Patient Demographics by FOG status. Means shown with standard deviation in parentheses. * indicates statistically significant difference between groups on an unpaired t-test. FOG- = People with Parkinson's disease without FOG; FOG+ = People with Parkinson's disease with FOG; H\&Y stage = Modified Hoehn \& Yahr stage; UPDRS III = Unified Parkinson's Disease Rating Scale III total; MOCA = Montreal Cognitive Assessment total; FAB = Frontal Assessment Battery total

\subsection{Behavioural data}

There was no significant difference in mean reaction times (time between stimulus presentation and button press response) between the ten PD without FOG (FOG-: blue) $(\mathrm{M}=546.0, \mathrm{SD}=72.95)$ and the ten with FOG (FOG+: grey) (M= 
562.2, $\mathrm{SD}=57.02)$ conditions; $(\mathrm{t}(18)=-0.5527, \mathrm{p}=0.58760$, JZS Bayes Factor $=2.25$ ). The JZS Bayes Factor analysis indicated that the null hypothesis (no difference in RTs between PD with and without FOG) was 2.25 times more likely than the alternative hypothesis. These means are shown in Figures 1 and 2 by vertical dashed lines. Similarly, there was no significant difference in the standard deviation of reaction times for FOG- $(\mathrm{M}=84.1, \mathrm{SD}=28.6)$ and FOG+ $(\mathrm{M}=86.4, \mathrm{SD}=24.53)$ conditions; $(\mathrm{t}(18)=-0.1967, \mathrm{p}=0.84$, JZS Bayes Factor $=2.482$ ).

\subsubsection{EEG Analysis: Cognitive Decision Making Potentials}

Figure 1 shows the mean and standard error of the mean (SEM) of the standard (green) and target (red) CSD response for both the FOG- group and FOG+ group (Figure 1) for three electrodes over central parietal scalp (blue dots). To assess difference in the amplitude of the P3/CPP, we submitted the mean amplitude response from $454 \mathrm{~ms}$ to $654 \mathrm{~ms}$ to a mixed repeated measures ANOVA with the factors group (FOG-, FOG+) and condition (target, standard). The analysis revealed a main effect of condition $(F(1,18)=34.332$, $M S E=5573.1, p<0.001$, JZS Bayes Factor $=0.001)$ with no effect of group $(F(1,18)=0.357$, MSE $=131.91$, $\mathrm{p}=0.55$, JZS Bayes Factor $=2.217$ ) or interaction of group and condition $(F(1,18)=0.505, M S E=81.99, p=0.486$, JZS Bayes Factor $=2.1)$. The JZS Bayes Factor analysis indicated that the null hypothesis (no difference in amplitude of the evoked potential between PD with and without FOG) was 2.22 times more likely than the alternative hypothesis. A more robust measure of this parameter is achieved by subtracting the response to the standard stimulus from the response to the target stimulus. The difference between target and standard responses for FOG- (blue) and FOG+ (grey) are shown in Figure 2 which shows no significant difference between groups $(\mathrm{t}(18)=-0.068, \mathrm{p}=0.95$, JZS Bayes Factor $=2.51$ ).

\subsubsection{EEG Analysis: Motor Preparation Potentials}

Figure 2 shows lateralized readiness potential CSD waveforms, the subtraction target response over left (green dots) and right (red dots) frontal areas, for the FOG- (blue) group and the FOG+ (grey) group. To investigate motor preparation differences between the groups the lateralized readiness potential (LRP) was calculated. The mean amplitude response from $454 \mathrm{~ms}$ to $654 \mathrm{~ms}$ was submitted to an unpaired t-test, the analysis revealed significant amplitude differences $(\mathrm{t}(18)=2.388, \mathrm{p}<0.05$, JZS Bayes Factor $=0.39988)$ between freezers (FOG + ) and non-freezers (FOG-). To investigate the onset of differences between groups in the LRP for each time point was submitted to an unpaired t-test. Time points of statistical differences in the LRP between the FOG+ group and the FOG-group are depicted as markers running along the bottom of the plot. The group difference in onset occurs just after $350 \mathrm{~ms}$ and continues until just before the mean response time indicated by the dashed vertical lines. 
Figure 2 also shows the CSD scalp distribution of the target response centered at $554 \mathrm{~ms}$ over $200 \mathrm{~ms}$ for the FOG- group (top) and FOG+ group (bottom). The distributions show clear positive peaks over central parietal scalp for both groups consistent with the CPP response. Over frontal sites there were also leftright lateralized differences consistent with a lateralized readiness potential which was more prominent in the FOG+ group than the FOG- group. The high spatial resolution of the CSD method allows these signals to be clearly identified and localized. For comparison, Figure 2C shows the scalp distribution for the target response using the standard ERP method for the FOG- group, top and FOG+ group, bottom. The distributions show clear positive peaks over central scalp consistent with the P3b response which was more prominent in the FOGgroup than the FOG+ group. Importantly, the frontal lateralized differences are obscured using the standard ERP method due the lower spatial resolution. The analysis presented here is replicated using a standard ERP approach in the Supplementary Figures for comparison. Of note, the ERP method suggests a significant difference in P3b amplitude between FOG+ and FOG-. This could be due to volume conduction from the frontal lateralized readiness potential. The CSD method employed here allows separation of these two distinct signals.

Place Figure 2 around here.

\subsection{Regression analysis}

Disease duration, Frontal Assessment Battery (FAB) score and gender differ significantly between FOG- and FOG+ (Table 1). To explore the relationship between the mean LRP from $454 \mathrm{~ms}$ to $654 \mathrm{~ms}$ and the FAB score taking disease duration into account, we performed a regression analysis on the entire PD cohort (Figure 3). The multiple linear regression was calculated to predict the LRP amplitude based on the patients total FAB score and disease duration. A significant regression equation was found $(F(2,17)=6.12, p<.01)$, with an $R^{2}$ of 0.419. The LRP predicted amplitude is equal to $-79.958+4.155$ (FAB) 0.178 (disease duration). Total FAB score was a significant predictor of LRP amplitude $(\mathrm{t}(19)=3.329, \mathrm{p}<0.005)$. Disease duration, however, was not a significant predictor of LRP amplitude $(\mathrm{p}=0.644)$. Furthermore, there was no significant regression between the FAB score and disease duration $(F(1,18)=$ $0.583, \mathrm{p}=0.455$, JZS Bayes Factor $=2.03581$ ), with an $\mathrm{R}^{2}$ of 0.031 . Separate regression analyses showed no correlation between LRP amplitude and markers of disease severity (Hoehn \& Yahr stage and Unified Parkinson's Disease Rating Scale III score). Thus LRP amplitude is not associated with overall motor performance in PD.

Place Figure 3 around here.

\section{Discussion:}


Freezing of gait is associated with deficits in perceptual, motor and executive dysfunction. The underlying pathophysiology remains incompletely understood. The standard ERP analysis (shown in the Supplementary Figures) suggests significant differences in P3b morphology between FOG- and FOG+. However better spatial resolution of CSD analysis reveals two distinct signals: a centroparietal positivity (equivalent to P3b) which is unaffected by FOG status; and a motor lateralized readiness potential (LRP) which occurs earlier with a greater (more negative) amplitude in FOG+ than in FOG-. These results will be discussed separately. These findings highlight the importance of cautious interpretation of ERP data in PD and show that motor preparation may be the primary deficit in FOG. These motor preparation differences occur even in the absence of any difference in motor performance (UPDRS III score and reaction time).

\subsection{Event-Related Potential Analysis:}

The most common method of analyzing neurophysiological responses to stimuli is event-related potential (ERP) analysis. The primary objective of our study was to examine differences in cortical markers of cognitive and motor function between FOG- and FOG+. Given the close association executive dysfunction and freezing, one would expect significant differences in cortical markers between groups. The CSD increases spatial resolution and shows two separate signals: a slow-rising negative potential in the frontal region (the lateralized readiness potential), associated with motor preparation(Shibasaki and Hallett, 2006); and a centroparietal positivity (CPP), the transformed equivalent of the P3b (Twomey et al., 2015).

The P3b is intricately linked with cognitive performance (Pelosi et al., 1992), especially to rapid allocation of attentional resources (Reinvang, 1999). The P3b is associated with context updating, stimulus classification and decision making in response to sensory stimuli (Twomey et al., 2015). These associations are relevant to FOG as FOG correlates with executive dysfunction, especially, divided attention and dual-tasking (Spildooren et al., 2010, Tard et al., 2015). Multiple studies have shown increased P3b latency in PD correlating with cognitive dysfunction (Goodin and Aminoff, 1987, O'Donnell et al., 1987, Toda et al., 1993, Bodis-Wollner et al., 1995, Katsarou et al., 2004, Matsui et al., 2007), disease severity (Silva Lopes et al., 2014) and impaired activity of daily living (Maeshima et al., 2002). However, our Bayes factor analysis of CSD data suggests that there is no difference in the P3b/CPP response between FOG- and FOG+, implying similar cognitive processing in decision-making among both groups. This is surprising considering the FOG- group had higher frontal executive (FAB) scores than the FOG+ group.

\subsection{Cognitive Decision Making Potentials:}

The standard (global reference) ERP topoplots in Figure 2C suggest that there is a difference in the spatial distribution of $\mathrm{P} 3 \mathrm{~b}$ between groups, with a more localized signal over the centroparietal area in FOG- and a more diffuse amplitude distribution in FOG+ extending into the right frontal area. The CSD topoplots (Figure 2B) shows that the P3b signal is composed of two separate signals: a centroparietal positivity (CPP) and the lateralized readiness potential. 
No difference in CPP morphology exists between groups. Volume conduction from this second negative signal and the choice of the global reference could result in an underestimation of the P3b amplitude in an ERP analysis and lead to apparent differences in P3b between people with PD and healthy controls (Verleger et al., 2013). This highlights the advantage of CSD analysis in PD to separate the signals and reduce volume conduction from frontal cortical activity which could lead to a possible misinterpretation of ERP results (Kelly and O'Connell, 2013). Motor potentials have previously been noted to interfere with P3b morphology during simple tasks such as a button press (Salisbury et al., 2001). To our knowledge, only one other study has employed CSD analysis in PD (van Wouwe et al., 2014). Standard ERP analysis has a lower spatial resolution than CSD analysis. The ERP analysis in the Supplementary Figures suggests that P3b amplitude is larger in FOG- than in FOG+ and incorrectly suggests differences in cognitive processing. Our CSD analysis allowed us to separate these two distinct signals elucidating a greater understanding of their roles.

\subsection{Motor Readiness Potentials:}

The second signal, the frontal negativity, is a readiness potential (or Bereitschaftpotential) which is defined as the cortical activity which precedes voluntary self-initiated movement (Shibasaki and Hallett, 2006). We analysed the readiness potential as a lateralized readiness potential from the CSD signal at a pair of standard frontocentral sites by subtracting the signal contralateral to the response from the signal ipsilateral to the response. The lateralized readiness potential is, therefore, a measure of unimanual motor readiness. Our results show the onset of the lateralised readiness potential is earlier and the resultant amplitude is greater in the FOG+ group (Figure 3), yet there is no difference in reaction times. This suggests that patients with FOG probably recruit more resources (probably from lateral premotor areas, as discussed below) in order to achieve the same reaction time as those without FOG. The correlations between cortical electrical potential amplitude and neuronal firing (Whittingstall and Logothetis, 2009), cortical thickness(Liem et al., 2012), cortical surface area (Elvsashagen et al., 2015) and the blood oxygen level dependent (BOLD) response (Zaehle et al., 2009) imply indirectly that the differences in LRP amplitude seen here are due a greater amount of cortex generating the response in that area. It is important to note that these differences in motor preparation are seen in the absence of any difference in overall baseline motor performance (UPDRS III score) between groups. This suggests that motor preparation occurs earlier and to a greater degree in FOG+ than FOG-, even when the task is not challenging. Furthermore, the amplitude of the lateralized readiness potential correlates strongly with total FAB scores (Figure 3), indicating a link between impairments in motor preparation and executive dysfunction in FOG.

\subsection{Motor preparation in PD:}

Initiation of movement is crucially dependent on the supplementary motor area (SMA). Given that the SMA receives significant dopaminergic input from the basal ganglia (via the thalamus), it is often postulated that known motor preparation deficits in PD arise primarily from SMA dysfunction (D'Ostilio et al., 2013). Motor readiness potentials have been studied in PD previously (Dick et 
al., 1989, Shibasaki and Hallett, 2006). Dick et al. recorded motor readiness potentials in PD patients off-medication during a simple motor task and showed that the very early component of the readiness potential (not recorded in our study) was reduced in the PD group but a later component (corresponding to the readiness potential discussed herein) was larger than in healthy controls (Dick et al., 1989). It was proposed that the reduced early component corresponded to SMA underactivity and that the compensatory augmentation of the later potential was due to overactivity in lateral premotor areas(Praamstra et al., 1996). Of note, people with PD initiate movement earlier in response to a visual cue than an internally generated volitional movement (Praamstra et al., 1996). This is achieved by initiating motor preparation in response to partial sensory information. As a result, lateralized readiness potentials in response to visual stimuli begin earlier in PD patients than in healthy controls (Praamstra et al., 1998) thus achieving reaction times comparable to healthy controls for cued motor tasks, but at the expense of a greater number of errors. Response selection and motor preparation occur concurrently and inhibition of a response post initiation pf motor preparation may be required. Deficits in such inhibitory control are common in PD (Obeso et al., 2011) and, especially, in patients with FOG (Vandenbossche et al., 2012). Hence, altered motor preparation occurs in PD via a compensatory shift from SMA activation to activation of a larger area of cortex including lateral premotor areas in order to facilitate movement. Of note deficient coupling between the lateral premotor areas, SMA and the primary motor cortex is reinstated by levodopa in PD (Herz et al., 2014).

\subsection{Motor preparation in FOG:}

Motor readiness potentials have not been studied in FOG to date. However, deficits in motor preparation have been a central hypothesis in the pathophysiology of FOG. Freezing commonly occurs at gait initiation and rhythmic knee trembling is often seen during freezing episodes. This may represent excessive anticipatory postural adjustments (fine adjustments in lower limb muscle groups which are integral in maintaining balance during movement preparation) due to compensation via altered SMA-mesencephalic connections (Jacobs et al., 2009). This is the basis for the decoupling model of FOG which proposes a dissociation between a pre-planned motor program and motor initiation, leading to a breakdown of controlled movement. Hence, SMA dysfunction is proposed to be central to FOG pathophysiology (Nutt et al., 2011). Functional MRI studies have shown reduced SMA activation in FOG+ while turning (Gilat et al., 2015) and structural and functional connectivity studies confirm altered connectivity between SMA and motor cortex (Canu et al., 2015) and between SMA and the subthalamic nucleus in FOG+(Fling et al., 2014). The differences in lateralized readiness potentials seen in the FOG+ group in our study may reflect excessive recruitment of lateral premotor areas to compensate for SMA dysfunction. Furthermore, Vandenbossche et al. showed that people with PD and FOG rely more on automatic response activations and hence, are less able to suppress automatic responses than non-freezers (Vandenbossche et al., 2012). Impairments in attentional set-shifting (Naismith et al., 2010, Shine et al., 2013b) and dual-tasking (Spildooren et al., 2010, Peterson et al., 2014) occur in FOG. Clearly, excess cortical and subcortical recruitment required to perform a simple task, makes inhibition of an undesired response difficult, and hinders 
rapid shifting between tasks or undertaking two tasks concurrently. Such limited flexibility of processing is seen PD and FOG (Shine et al., 2013a) but also in healthy older adults (Malcolm et al., 2015). Dual-tasking in healthy subjects requires activation of extensive cortical networks which include the SMA and premotor areas (Sigman and Dehaene, 2008). When dual-tasking in PD is compared to healthy controls, greater activation of multiple cortical areas, including premotor areas, is required (Wu and Hallett, 2008). These areas are involved in simple motor preparation, it is likely that excessive interference will occur. Excessive activation during movement in FOG + has also been shown in imaging studies (Fasano et al., 2015). fMRI studies reveal increased activation within frontoparietal cortical regions during freezing of gait (Shine et al., 2013a) or freezing of upper limb movements (Vercruysse et al., 2014). However, experiments with complex or bimanual motor tasks have revealed these changes (Peterson et al., 2014). Our results show that these responses occur even with simple motor tasks such as a button press.

\subsection{Information overload:}

As mentioned above, Twomey et al. have recently proposed that the P3b (and by extension, the centroparietal positivity) represents a decision variable in response to information accumulation from sensory stimuli building to a threshold when a response is executed (Twomey et al., 2015). Moreover, the rate of this build-to-threshold determines the speed of response. However, the lateralized readiness potential has similar build-to-threshold dynamics and interacts with the CPP (Kelly and O'Connell, 2013). Thus, both CPP and LRP build in response to presented sensory information before a motor response (such as a button press) is triggered. Such a threshold concept is an attractive model given that a "sequence effect" is often observed in people with PD and FOG (Iansek et al., 2006, Chee et al., 2009) whereby gradual scaling of motor output is observed until a threshold is reached below which freezing occurs. This threshold model of FOG (Plotnik et al., 2012) can be demonstrated in upper limb movements of freezers (Vercruysse et al., 2012) and can be used to trigger freezing with rapid small steps or stepping in place(Snijders et al., 2008).

The motor task used here is a simple one. During more complex tasks such as locomotion it is likely that excessive recruitment would require extensive attentional resources in order to walk through a doorway and could lead to breakdown of motor function. Increased (and possibly disorganized) compensatory motor readiness could lead to significant interference, especially in the face of a competing cognitive/motor task or a complex sensory environment. The neural reserve (interference) model of FOG, proposed by Lewis and Barker, formulates FOG as a breakdown of processing of concurrent motor, cognitive and limbic inputs through a deficient basal ganglia with a smaller capacity for parallel processing (Lewis and Barker, 2009). Recently, Beck et al. examined FOG while walking towards a doorway and concluded that FOG may be the result of an overload of cognitive and sensory information (Beck et al., 2015). Our findings show explicitly that excessive motor processing occurs upstream at the cortical level, leading to a greater amount of information for processing. This effect is likely to be exaggerated by multiple cognitive tasks or complex sensory inputs. 
Both executive dysfunction and motor preparation are thought to be central to FOG pathophysiology, however few studies have linked these two entities. People with PD progressively lose automatic (habitual) control of movement. This can be compensated for by recruiting frontal networks leading to an overreliance on goal-directed motor control. It has previously been suggested that the apparent executive function deficits seen in PD could be due to overloading these frontal networks in the setting of loss of automatic motor control (Redgrave et al., 2010). We have shown that as executive function worsens the lateralized readiness potential enlarges. Thus, the aberrant motor preparation in FOG may require both loss of basal ganglia-SMA connectivity and frontal executive dysfunction. Alternatively, the loss of automaticity in PD and the resultant reliance on goal-directed control could lead to an overload on frontal processing mechanisms causing a secondary apparent impairment in executive function (rather than a primary deficit in executive function). Either way, the correlation between the lateralized readiness potential amplitude and FAB scores suggest that altered cortical motor preparation coincides with the appearance of executive dysfunction in PD, (although a causative association cannot be demonstrated in the current study). However, it is likely that any superimposed executive dysfunction in PD would stress these limited resources further, increasing the likelihood of motor breakdown in conflict or dual-task situations, resulting in FOG.

We have previously shown relative sensory processing differences in PD which correlated with disease duration and FOG status (Fearon et al., 2015). Here, we have described a marker of differences in motor preparation with respect to FOG status even in the absence of differences in standard clinical measures of motor processing (reaction time and UPDRS). Taken together our findings strive to explore sensitive and subtle sensory and motor biomarkers of PD and FOG for early intervention, even possibly in the preclinical phase of the disease.

\subsection{Limitations and Future Work:}

The sample size in the current study is small and the gender imbalance between groups may have contributed significantly to the results. Future work should include examining the effect of dopaminergic therapy on the above findings. All patients were tested in the "on"-medication state. Although there were no differences in medication doses or timings between groups, it would be necessary to confirm these findings off medication. The task used in this study is simple, and not directly related to gait. However, the findings highlight abnormal movement preparation, even for a simple movement tasks. It is likely that these deficits are also present for more complex tasks such as gait but this should be confirmed with further studies. In addition, future work should consider the effect of deep brain stimulation on these parameters as this may shed light on why stimulation can relieve FOG in some patients and induce it in others. This paradigm could also be used to explore other disease cohorts such as patients with progressive supranuclear palsy and vascular parkinsonism in whom FOG and cognitive dysfunction occur earlier and are more common. Finally, the lateralised readiness potential could be a potential biomarker for predicting 
those PD patients that will ultimately develop FOG as well as a metric for response to interventions for freezing.

\section{Conclusion:}

In summary, these results suggest that no difference in centroparietal positivity morphology exists between FOG+ and FOG-, implying that decision making and reaction time in response to sensory information is equivalent in both groups. However, motor preparation occurs earlier and requires greater recruitment in FOG+ suggesting that this may be the primary deficit in FOG. These motor preparation differences occur even when overall motor performance is equivalent but probably overload frontal networks during more complex tasks. There is a significant difference in FAB scores between FOG+ and FOG-, which correlates strongly with the amplitude of the lateralized readiness potential, highlighting the important interaction of executive dysfunction and motor preparation in the evolution of FOG.

\section{Conflict of interest:}

None of the authors have potential conflicts of interest to be disclosed.

\section{Acknowledgments:}

The authors would like to thank the Trinity Centre for Bioengineering team, the Dublin Neurological Institute at the Mater Misericordiae Hospital, and the VERVE FP7 Project. They would also like to thank all the participants who took part in this study. 


\section{References:}

Amboni M, Cozzolino A, Longo K, Picillo M, Barone P. Freezing of gait and executive functions in patients with Parkinson's disease. Mov Disord. 2008;23:395-400, 10.1002/mds.21850.

Beck EN, Ehgoetz Martens KA, Almeida QJ. Freezing of Gait in Parkinson's Disease: An Overload Problem? PLoS One. 2015;10:e0144986, 10.1371/journal.pone.0144986.

Bloem BR, Hausdorff JM, Visser JE, Giladi N. Falls and freezing of gait in Parkinson's disease: a review of two interconnected, episodic phenomena. Mov Disord. 2004;19:871-84, 10.1002/mds.20115.

Bodis-Wollner I, Borod JC, Cicero B, Haywood CS, Raskin S, Mylin L, et al. Modality dependent changes in event-related potentials correlate with specific cognitive functions in nondemented patients with Parkinson's disease. J Neural Transm Park Dis Dement Sect. 1995;9:197-209.

Canu E, Agosta F, Sarasso E, Volonte MA, Basaia S, Stojkovic T, et al. Brain structural and functional connectivity in Parkinson's disease with freezing of gait. Hum Brain Mapp. 2015;36:5064-78, 10.1002/hbm.22994.

Chee R, Murphy A, Danoudis M, Georgiou-Karistianis N, Iansek R. Gait freezing in Parkinson's disease and the stride length sequence effect interaction. Brain. 2009;132:2151-60, 10.1093/brain/awp053.

Cohen RG, Klein KA, Nomura M, Fleming M, Mancini M, Giladi N, et al. Inhibition, executive function, and freezing of gait. J Parkinsons Dis. 2014;4:111-22, 10.3233/JPD-130221.

Cunnington R, Iansek R, Bradshaw JL, Phillips JG. Movement-related potentials in Parkinson's disease. Presence and predictability of temporal and spatial cues. Brain. 1995;118:935-50.

D'Ostilio K, Deville B, Cremers J, Grandjean J, Skawiniak E, Delvaux V, et al. Role of the supplementary motor area in the automatic activation of motor plans in de novo Parkinson's disease patients. Neurosci Res. 2013;76:173-7, 10.1016/j.neures.2013.04.002.

Dick JP, Rothwell JC, Day BL, Cantello R, Buruma 0, Gioux M, et al. The Bereitschaftspotential is abnormal in Parkinson's disease. Brain. 1989;112:23344.

Dienes Z. How Bayes factors change scientific practice. J Math Psychol. 2016;72:78-89, http://dx.doi.org/10.1016/j.jmp.2015.10.003.

Elvsashagen T, Moberget T, Boen E, Hol PK, Malt UF, Andersson S, et al. The surface area of early visual cortex predicts the amplitude of the visual evoked potential. Brain Struct Funct. 2015;220:1229-36, 10.1007/s00429-013-0703-7. Fasano A, Herman T, Tessitore A, Strafella AP, Bohnen NI. Neuroimaging of Freezing of Gait. J Parkinsons Dis. 2015;5:241-54, 10.3233/JPD-150536. Fearon C, Butler JS, Newman L, Lynch T, Reilly RB. Audiovisual Processing is Abnormal in Parkinson's Disease and Correlates with Freezing of Gait and Disease Duration. J Parkinsons Dis. 2015;5:925-36, 10.3233/JPD-150655. Fling BW, Cohen RG, Mancini M, Carpenter SD, Fair DA, Nutt JG, et al. Functional reorganization of the locomotor network in Parkinson patients with freezing of gait. PLoS One. 2014;9:e100291, 10.1371/journal.pone.0100291. 
Georgiev D, Lange F, Seer C, Kopp B, Jahanshahi M. Movement-related potentials in Parkinson's disease. Clin Neurophysiol. 2016;127:2509-19, 10.1016/j.clinph.2016.04.004.

Giladi N, Nieuwboer A. Understanding and treating freezing of gait in parkinsonism, proposed working definition, and setting the stage. Mov Disord. 2008;23 Suppl 2:S423-5, 10.1002/mds.21927.

Gilat M, Shine JM, Walton CC, O'Callaghan C, Hall JM, Lewis SJ. Brain activation underlying turning in Parkinson's disease patients with and without freezing of gait: a virtual reality fMRI study. NPJ Parkinson's Dis. 2015;1:15020.

Goodin DS, Aminoff MJ. Electrophysiological differences between demented and nondemented patients with Parkinson's disease. Ann Neurol. 1987;21:90-4,

10.1002/ana.410210116.

Groom MJ, Cragg L. Differential modulation of the N2 and P3 event-related potentials by response conflict and inhibition. Brain Cogn. 2015;97:1-9, 10.1016/j.bandc.2015.04.004. Guthrie D, Buchwald JS. Significance testing of difference potentials.

Psychophysiology. 1991;28:240-4.

Handojoseno AM, Shine JM, Nguyen TN, Tran Y, Lewis SJ, Nguyen HT. The detection of Freezing of Gait in Parkinson's disease patients using EEG signals based on Wavelet decomposition. Conf Proc IEEE Eng Med Biol Soc. 2012;2012:69-72, 10.1109/EMBC.2012.6345873.

Handojoseno AM, Shine JM, Nguyen TN, Tran Y, Lewis SJ, Nguyen HT. Using EEG spatial correlation, cross frequency energy, and wavelet coefficients for the prediction of Freezing of Gait in Parkinson's Disease patients. Conf Proc IEEE Eng Med Biol Soc. 2013;2013:4263-6, 10.1109/EMBC.2013.6610487.

Herz DM, Florin E, Christensen MS, Reck C, Barbe MT, Tscheuschler MK, et al. Dopamine replacement modulates oscillatory coupling between premotor and motor cortical areas in Parkinson's disease. Cereb Cortex. 2014;24:2873-83, 10.1093/cercor/bht140.

Hughes AJ, Daniel SE, Kilford L, Lees AJ. Accuracy of clinical diagnosis of idiopathic Parkinson's disease: a clinico-pathological study of 100 cases. J Neurol Neurosurg Psychiatry. 1992;55:181-4.

Iansek R, Huxham F, McGinley J. The sequence effect and gait festination in Parkinson disease: contributors to freezing of gait? Mov Disord. 2006;21:141924, 10.1002/mds.20998.

Jacobs JV, Nutt JG, Carlson-Kuhta P, Stephens M, Horak FB. Knee trembling during freezing of gait represents multiple anticipatory postural adjustments. Exp Neurol. 2009;215:334-41, 10.1016/j.expneurol.2008.10.019. Jahanshahi M, Jenkins IH, Brown RG, Marsden CD, Passingham RE, Brooks DJ. Self-initiated versus externally triggered movements. I. An investigation using measurement of regional cerebral blood flow with PET and movement-related potentials in normal and Parkinson's disease subjects. Brain. 1995;118:913-33. Katsarou Z, Bostantjopoulou S, Kimiskidis V, Rossopoulos E, Kazis A. Auditory event-related potentials in Parkinson's disease in relation to cognitive ability. Percept Mot Skills. 2004;98:1441-8, 10.2466/pms.98.3c.1441-1448.

Kayser J, Tenke CE. Principal components analysis of Laplacian waveforms as a generic method for identifying ERP generator patterns: II. Adequacy of lowdensity estimates. Clin Neurophysiol. 2006;117:369-80, 10.1016/j.clinph.2005.08.033. 
Kelly SP, O'Connell RG. Internal and external influences on the rate of sensory evidence accumulation in the human brain. J Neurosci. 2013;33:19434-41, 10.1523/JNEUROSCI.3355-13.2013.

Killane I, Fearon C, Newman L, McDonnell C, Waechter SM, Sons K, et al. Dual Motor-Cognitive Virtual Reality Training Impacts Dual-Task Performance in Freezing of Gait. IEEE J Biomed Health Inform. 2015;19:1855-61, 10.1109/JBHI.2015.2479625.

Kindermann SS, Kalayam B, Brown GG, Burdick KE, Alexopoulos GS. Executive functions and P300 latency in elderly depressed patients and control subjects. Am J Geriatr Psychiatry. 2000;8:57-65.

Lewis SJ, Barker RA. A pathophysiological model of freezing of gait in Parkinson's disease. Parkinsonism Relat Disord. 2009;15:333-8, 10.1016/j.parkreldis.2008.08.006.

Liem F, Zaehle T, Burkhard A, Jancke L, Meyer M. Cortical thickness of supratemporal plane predicts auditory N1 amplitude. Neuroreport. 2012;23:1026-30, 10.1097/WNR.0b013e32835abc5c.

Maeshima S, Itakura T, Komai N, Matsumoto T, Ueyoshi A. Relationships between event-related potentials (P300) and activities of daily living in Parkinson's disease. Brain Inj. 2002;16:1-8, 10.1080/02699050110088245.

Malcolm BR, Foxe JJ, Butler JS, De Sanctis P. The aging brain shows less flexible reallocation of cognitive resources during dual-task walking: A mobile brain/body imaging (MoBI) study. Neuroimage. 2015;117:230-42, 10.1016/j.neuroimage.2015.05.028. Maruyama T, Yanagisawa N. Cognitive impact on freezing of gait in Parkinson's disease. Parkinsonism Relat Disord. 2006;12:S77-S82.

Matsui H, Nishinaka K, Oda M, Kubori T, Udaka F. Auditory event-related potentials in Parkinson's disease: prominent correlation with attention. Parkinsonism Relat Disord. 2007;13:394-8, 10.1016/j.parkreldis.2006.12.012. Naismith SL, Shine JM, Lewis SJ. The specific contributions of set-shifting to freezing of gait in Parkinson's disease. Mov Disord. 2010;25:1000-4, $10.1002 /$ mds.23005.

Nieuwboer A, Giladi N. Characterizing freezing of gait in Parkinson's disease: models of an episodic phenomenon. Mov Disord. 2013;28:1509-19, $10.1002 /$ mds. 25683.

Nieuwboer A, Herman T, Rochester L, Emil GE, Giladi N. P2. 097a The new revised freezing of gait questionnaire, a reliable and valid instrument to measure freezing in Parkinson's disease? Parkinsonism Relat Disord. 2008;14:S68.

Nutt JG, Bloem BR, Giladi N, Hallett M, Horak FB, Nieuwboer A. Freezing of gait: moving forward on a mysterious clinical phenomenon. Lancet Neurol. 2011;10:734-44, 10.1016/S1474-4422(11)70143-0.

O'Connell RG, Dockree PM, Kelly SP. A supramodal accumulation-to-bound signal that determines perceptual decisions in humans. Nat Neurosci. 2012;15:1729$35,10.1038 / \mathrm{nn} .3248$.

O'Donnell BF, Squires NK, Martz MJ, Chen JR, Phay AJ. Evoked potential changes and neuropsychological performance in Parkinson's disease. Biol Psychol. 1987;24:23-37.

Obeso I, Wilkinson L, Casabona E, Bringas ML, Alvarez M, Alvarez L, et al. Deficits in inhibitory control and conflict resolution on cognitive and motor tasks in 
Parkinson's disease. Exp Brain Res. 2011;212:371-84, 10.1007/s00221-0112736-6.

Pelosi L, Holly M, Slade T, Hayward M, Barrett G, Blumhardt LD. Event-related potential (ERP) correlates of performance of intelligence tests.

Electroencephalogr Clin Neurophysiol. 1992;84:515-20.

Perrin F, Pernier J, Bertrand 0, Echallier JF. Spherical Splines for Scalp Potential and Current-Density Mapping. Electroencephalogr Clin Neurophysiol. 1989;72:184-7.

Peterson DS, Pickett KA, Duncan R, Perlmutter J, Earhart GM. Gait-related brain activity in people with Parkinson disease with freezing of gait. PLoS One. 2014;9:e90634, 10.1371/journal.pone.0090634.

Plotnik M, Giladi N, Hausdorff JM. Is freezing of gait in Parkinson's disease a result of multiple gait impairments? Implications for treatment. Parkinsons Dis. 2012;2012:459321, 10.1155/2012/459321.

Polich J. Updating P300: an integrative theory of P3a and P3b. Clin Neurophysiol. 2007;118:2128-48, 10.1016/j.clinph.2007.04.019.

Praamstra P, Meyer AS, Cools AR, Horstink MW, Stegeman DF. Movement preparation in Parkinson's disease. Time course and distribution of movementrelated potentials in a movement precueing task. Brain. 1996;119:1689-704.

Praamstra P, Stegeman DF, Cools AR, Horstink MW. Reliance on external cues for movement initiation in Parkinson's disease. Evidence from movement-related potentials. Brain. 1998;121:167-77.

Redgrave P, Rodriguez M, Smith Y, Rodriguez-Oroz MC, Lehericy S, Bergman H, et al. Goal-directed and habitual control in the basal ganglia: implications for Parkinson's disease. Nat Rev Neurosci. 2010;11:760-72, 10.1038/nrn2915.

Reinvang I. Cognitive event-related potentials in neuropsychological assessment. Neuropsychol Rev. 1999;9:231-48.

Rouder JN, Speckman PL, Sun D, Morey RD, Iverson G. Bayesian t tests for accepting and rejecting the null hypothesis. Psychon Bull Rev. 2009;16:225-37, 10.3758/PBR.16.2.225.

Salisbury DF, Rutherford B, Shenton ME, McCarley RW. Button-pressing affects P300 amplitude and scalp topography. Clin Neurophysiol. 2001;112:1676-84. Shibasaki H, Hallett M. What is the Bereitschaftspotential? Clin Neurophysiol. 2006;117:2341-56, 10.1016/j.clinph.2006.04.025.

Shine JM, Handojoseno AM, Nguyen TN, Tran Y, Naismith SL, Nguyen H, et al. Abnormal patterns of theta frequency oscillations during the temporal evolution of freezing of gait in Parkinson's disease. Clin Neurophysiol. 2014;125:569-76, 10.1016/j.clinph.2013.09.006.

Shine JM, Matar E, Ward PB, Bolitho SJ, Gilat M, Pearson M, et al. Exploring the cortical and subcortical functional magnetic resonance imaging changes associated with freezing in Parkinson's disease. Brain. 2013a;136:1204-15, 10.1093/brain/awt049.

Shine JM, Naismith SL, Palavra NC, Lewis SJ, Moore ST, Dilda V, et al. Attentional set-shifting deficits correlate with the severity of freezing of gait in Parkinson's disease. Parkinsonism Relat Disord. 2013b;19:388-90, 10.1016/j.parkreldis.2012.07.015.

Sigman M, Dehaene S. Brain mechanisms of serial and parallel processing during dual-task performance. J Neurosci. 2008;28:7585-98, 10.1523/JNEUROSCI.094808.2008 . 
Silva Lopes M, Souza Melo A, Nobrega AC. Delayed latencies of auditory evoked potential P300 are associated with the severity of Parkinson's disease in older patients. Arq Neuropsiquiatr. 2014;72:296-300.

Singh A, Plate A, Kammermeier S, Mehrkens JH, Ilmberger J, Bötzel K. Freezing of gait-related oscillatory activity in the human subthalamic nucleus. Basal Ganglia. 2013;3:25-32.

Snijders AH, Nijkrake MJ, Bakker M, Munneke M, Wind C, Bloem BR. Clinimetrics of freezing of gait. Mov Disord. 2008;23 Suppl 2:S468-74, 10.1002/mds.22144.

Spildooren J, Vercruysse S, Desloovere K, Vandenberghe W, Kerckhofs E,

Nieuwboer A. Freezing of gait in Parkinson's disease: the impact of dual-tasking and turning. Mov Disord. 2010;25:2563-70, 10.1002/mds.23327.

Sutton S, Braren M, Zubin J, John ER. Evoked-potential correlates of stimulus uncertainty. Science. 1965;150:1187-8.

Tard C, Delval A, Duhamel A, Moreau C, Devos D, Dujardin K. Specific Attentional Disorders and Freezing of Gait in Parkinson's Disease. J Parkinsons Dis.

2015;5:379-87, 10.3233/JPD-140498.

Tard C, Dujardin K, Bourriez JL, Destee A, Derambure P, Defebvre L, et al. Attention modulates step initiation postural adjustments in Parkinson freezers. Parkinsonism Relat Disord. 2014;20:284-9, 10.1016/j.parkreldis.2013.11.016. Thevathasan W, Pogosyan A, Hyam JA, Jenkinson N, Foltynie T, Limousin P, et al. Alpha oscillations in the pedunculopontine nucleus correlate with gait performance in parkinsonism. Brain. 2012;135:148-60, 10.1093/brain/awr315. Toda K, Tachibana H, Sugita M, Konishi K. P300 and reaction time in Parkinson's disease. J Geriatr Psychiatry Neurol. 1993;6:131-6.

Toledo JB, Lopez-Azcarate J, Garcia-Garcia D, Guridi J, Valencia M, Artieda J, et al. High beta activity in the subthalamic nucleus and freezing of gait in Parkinson's disease. Neurobiol Dis. 2014;64:60-5, 10.1016/j.nbd.2013.12.005.

Twomey DM, Murphy PR, Kelly SP, O'Connell RG. The classic P300 encodes a build-to-threshold decision variable. Eur J Neurosci. 2015;42:1636-43, 10.1111/ejn.12936. van Wouwe NC, van den Wildenberg WP, Claassen DO, Kanoff K, Bashore TR, Wylie SA. Speed pressure in conflict situations impedes inhibitory action control in Parkinson's disease. Biol Psychol. 2014;101:44-60,

10.1016/j.biopsycho.2014.07.002.

Vandenbossche J, Deroost N, Soetens E, Zeischka P, Spildooren J, Vercruysse S, et al. Conflict and freezing of gait in Parkinson's disease: support for a response control deficit. Neuroscience. 2012;206:144-54, 10.1016/j.neuroscience.2011.12.048.

Velu PD, Mullen T, Noh E, Valdivia MC, Poizner H, Baram Y, et al. Effect of visual feedback on the occipital-parietal-motor network in Parkinson's disease with freezing of gait. Front Neurol. 2014;4:209, 10.3389/fneur.2013.00209.

Vercruysse S, Spildooren J, Heremans E, Vandenbossche J, Wenderoth N, Swinnen SP, et al. Abnormalities and cue dependence of rhythmical upper-limb movements in Parkinson patients with freezing of gait. Neurorehabil Neural Repair. 2012;26:636-45, 10.1177/1545968311431964.

Vercruysse S, Spildooren J, Heremans E, Wenderoth N, Swinnen SP, Vandenberghe $\mathrm{W}$, et al. The neural correlates of upper limb motor blocks in Parkinson's disease and their relation to freezing of gait. Cereb Cortex. 2014;24:3154-66, 10.1093/cercor/bht170. 
Verleger R, Schroll H, Hamker FH. The unstable bridge from stimulus processing to correct responding in Parkinson's disease. Neuropsychologia. 2013;51:251225, 10.1016/j.neuropsychologia.2013.09.017.

Whittingstall K, Logothetis NK. Frequency-band coupling in surface EEG reflects spiking activity in monkey visual cortex. Neuron. 2009;64:281-9, 10.1016/j.neuron.2009.08.016.

$\mathrm{Wu} \mathrm{T}$, Hallett M. Neural correlates of dual task performance in patients with Parkinson's disease. J Neurol Neurosurg Psychiatry. 2008;79:760-6, 10.1136/jnnp.2007.126599.

Zaehle T, Frund I, Schadow J, Tharig S, Schoenfeld MA, Herrmann CS. Inter- and intra-individual covariations of hemodynamic and oscillatory gamma responses in the human cortex. Front Hum Neurosci. 2009;3:8, 10.3389/neuro.09.008.2009. 


\section{Figure Captions}

Figure 1. Decision Making. The mean and standard error of the mean of the target (red) and standard (green) average CSD response of three electrodes (corresponding to A2, A3 and A4 in the 128 Biosemi ABC electrode layout) over central parietal scalp (indicated by the blue dots in the top down head schematic) for A. the FOG- group and B. the FOG+ group. The solid black line indicates the stimulus onset, the dashed vertical lines indicate the mean response time for the FOG- (blue) group and FOG+ (grey) group. FOG- = People with Parkinson's disease without FOG; FOG+ = People with Parkinson's disease with FOG.

Figure 2. Motor preparation and decision making. A. Upper plot: Mean and standard error of the mean of the lateralized readiness potential current source density (CSD) calculated by subtracting the average activity of three electrodes over the left frontocentral area (three green electrodes corresponding to D3, D4 and $\mathrm{D} 5$ in the 128 Biosemi $\mathrm{ABC}$ electrode layout) from the right frontocentral (three red electrodes corresponding to C3, C4 and C5 in the 128 Biosemi ABC electrode layout) area for the FOG- (blue) group and FOG+ (grey) group. Lower plot: Mean and standard error of the mean of the difference between the CSD waveform for the target stimulus (rotated green cross presented for $500 \mathrm{msecs}$ ) and standard stimulus (vertical green cross presented for $500 \mathrm{msecs}$ ) over central parietal scalp (blue dots) for the FOG- (blue) group and FOG+ (grey) group. The solid black line indicates the stimulus onset, the dashed vertical lines indicate the mean response time for the FOG- (blue) group and FOG+ (grey) group. The dots at the bottom of the graph indicate individual time points of statistically significant differences between the groups in the LRP waveform. B. The mean CSD scalp distribution for the FOG- (top) group and FOG+ (bottom) group. C. The mean scalp distribution for the FOG- (top) group and FOG+ (bottom) group using the standard event-related potential (ERP) method for comparison. FOG- = People with Parkinson's disease without FOG; FOG+ = People with Parkinson's disease with FOG.

Figure 3. Relationship of lateralized readiness potential (LRP) amplitude and frontal assessment battery (FAB) score. Scatterplot displays on the x-axis FAB score and on the y-axis the mean amplitude of the LRP from $454 \mathrm{~ms}$ to $654 \mathrm{~ms}$. Each circle represents a person with Parkinson's disease, the solid line indicates the significant regression fit for the data. 


\section{Motor Preparation Rather Than Decision-Making Differentiates Parkinson's Disease Patients With And Without Freezing of Gait}

Objective: Freezing of gait (FOG) is a brief, episodic phenomenon affecting over half of people with Parkinson's disease (PD) and leads to significant morbidity. The pathophysiology of FOG remains poorly understood but is associated with deficits in cognitive function and motor preparation.

Method: We studied 20 people with PD (10 with FOG, 10 without FOG) and performed a timed response target detection task while electroencephalographic data were acquired. We analysed the data to detect and examine cortical markers of cognitive decision making (P3b or centroparietal positivity, CPP) and motor readiness potential. We analysed current source density (CSD) to increase spatial resolution and allow identification of distinct signals.

Results: There was no difference in the $\mathrm{P} 3 \mathrm{~b} / \mathrm{CPP}$ response between people with PD with and without FOG, suggesting equivalent cognitive processing with respect to decision-making. However, the FOG group had significant difference with an earlier onset and larger amplitude of the lateralized readiness potential. Furthermore, the amplitude of the lateralised readiness potential correlated strongly with total Frontal Assessment Battery score.

Conclusions: The difference in lateralized readiness potentials may reflect excessive recruitment of lateral premotor areas to compensate for dysfunction of the supplementary motor area and resultant loss of automatic motor control. This early, excessive recruitment of frontal networks occurs in spite of equivalent motor scores and reaction times between groups.

Significance: The saturation of frontal processing mechanisms could help explain deficits in attentional set-shifting, dual-tasking and response inhibition which are frequently reported in FOG. 
A) FOG-

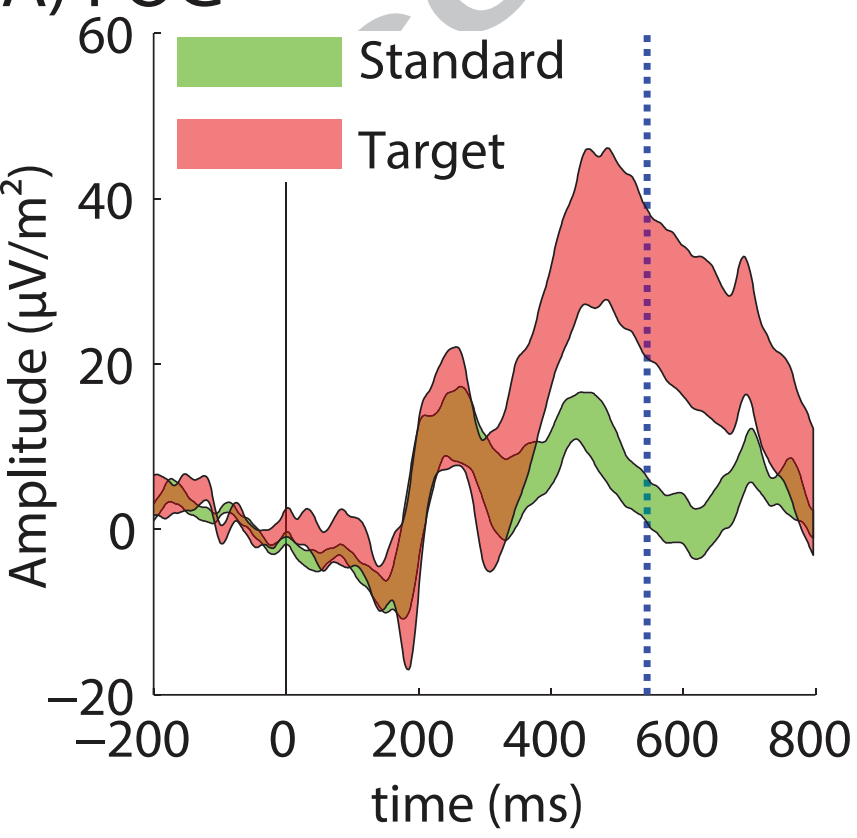

B) $\mathrm{FOG}+$

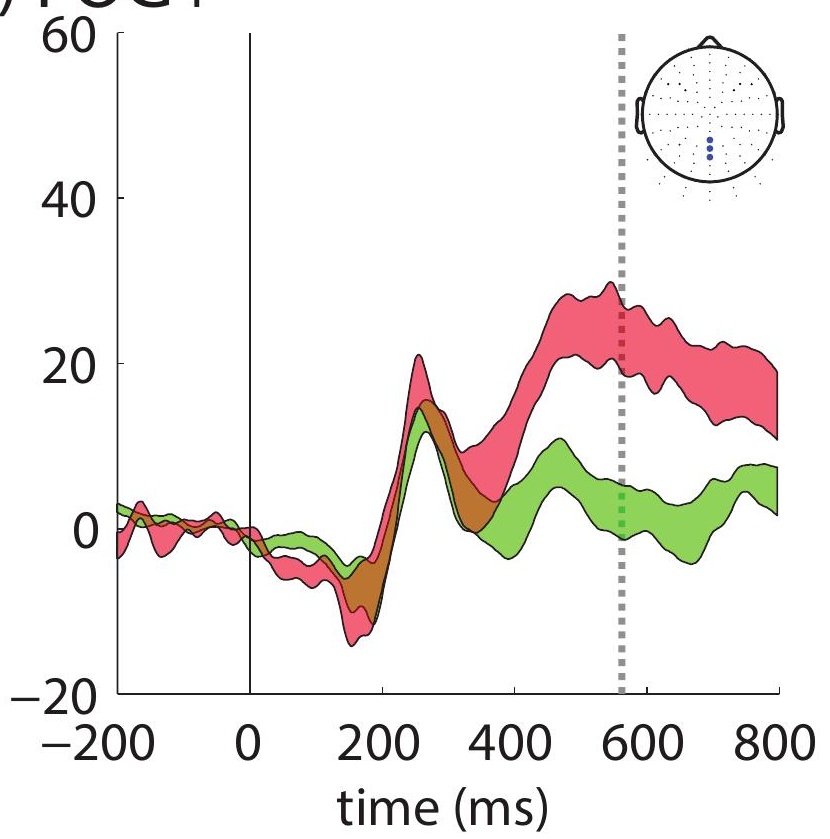


Lateralised Readiness Potential (LRP)

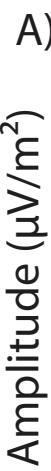

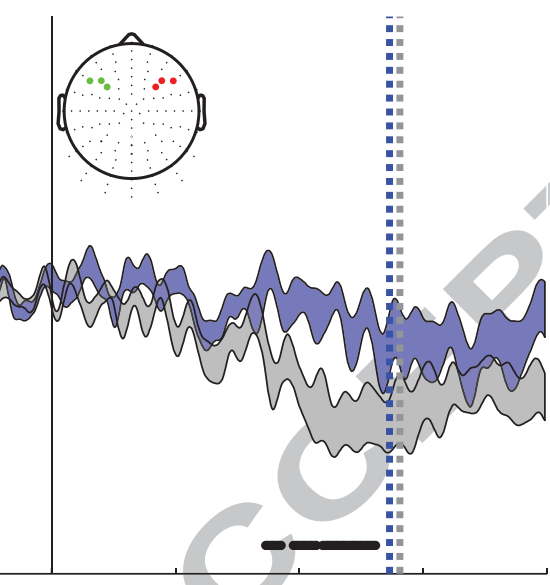

Target - Standard

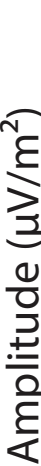

\begin{tabular}{l|l} 
B) CSD & C) ERP
\end{tabular}

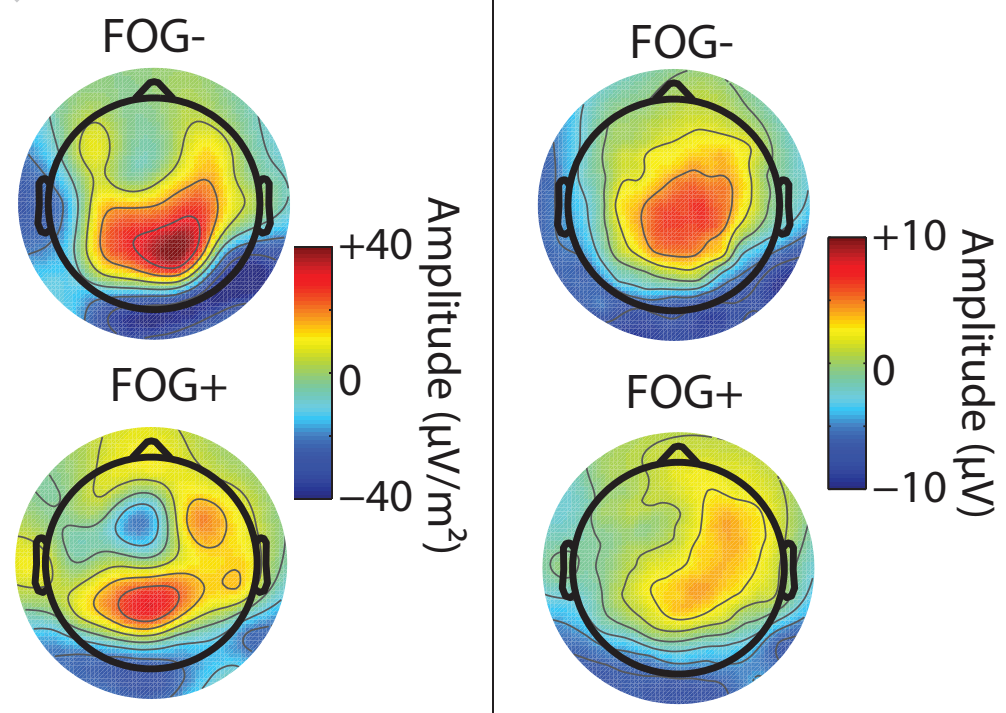

454-654ms

454-654ms 


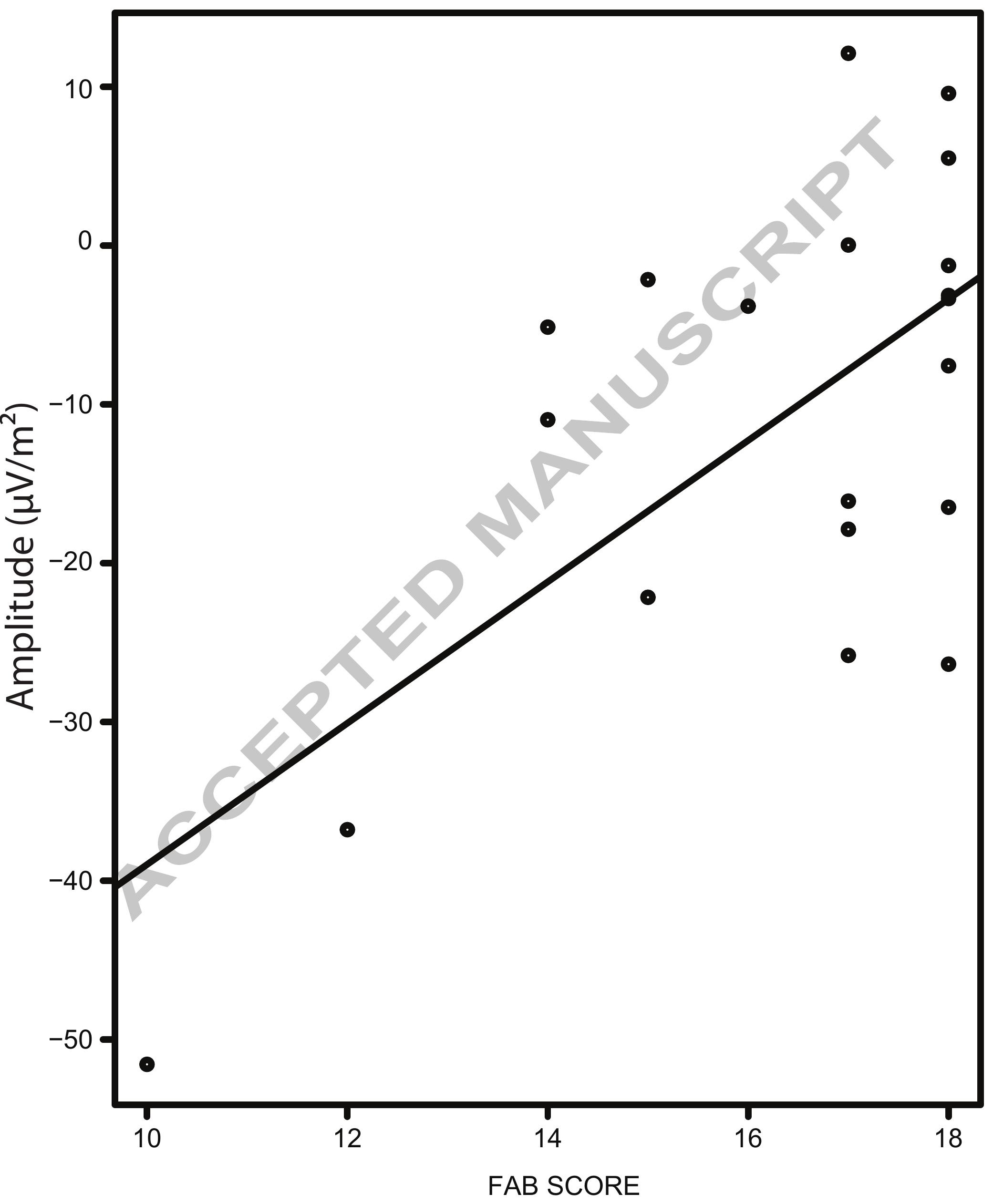

